DOI: $10.2478 /$ lpts-2021-0035

\title{
MODELLING OF DRAG FORCE REDUCTION FOR A WATERJET PROPULSION SYSTEM
}

\author{
M. Cerpinska*, M. Irbe, A. Pupurs, K. Burbeckis \\ Laboratory of Experimental Mechanics of Materials, Riga Technical University, \\ 6B-525 Kipsalas Str., Riga, LV-1048, LATVIA \\ *e-mail: marina.cerpinska@rtu.Iv
}

The paper provides simulation results for SUP (Stand Up Paddle) board appendage resistance. Additional propulsion is added to the SUP board. It is equipped with a waterjet. The waterjet is attached to the board rudder. This increases the drag coefficient for rudder five times. To reduce the drag variable, design options for the waterjet duct were proposed. The simulation tests were performed using SolidWorks Flow software using two types of simulations, namely, the pressure on the body and the flow around the body. The objective was to streamline the bluff duct of the waterjet and thus to create the appendage design with minimum drag force from fluid flow and possibly greater Inlet Velocity Ratio. Calculations showed that rounding-off the edges of waterjet duct resulted in $35 \%$ of drag coefficient reduction, while further streamlining reduced it by additional $10 \%$.

Keywords: Drag coefficient, propulsion, resistance, SUP board, waterjet.

\section{INTRODUCTION}

This study is the first part of a project aimed at designing the optimal waterjet to motorise the SUP (Stand Up Paddle) board.

The idea of motorising SUP boards for leisure activities is lucrative for two reasons. First, it provides existing users with new experience. It allows relaxing on the water and organising adventurous board trips for longer distances. Second, it attracts new users previously not interested in SUP boarding due to the slow motion of the board or the necessity to paddle actively to get propulsion.

Motorising the leisure activity brings in the dilemma, as discussed in a book by Jennings devoted to Motorised Water Sports [1]. 
It is necessary to consider damage to the local environment. Yet, the author stresses that motorised boards provide a lot of positive emotions, broadens tourism offerings and, as a result, provide economic benefits for local and regional community [1].

The goal of the project is to attach a waterjet to the SUP fin (rudder). There could be three fins installed on one board, but the design with one fin is more usual. This project involves two teams working together. The team of electrical engineers is responsible for the selection of motor and control system for propulsion. The team of mechanical engineers is responsible for the design of propulsion components. The first challenge for the mechanical engineers is to evaluate the hydrodynamic resistance of the attached object and to choose a design with least resistance because unmodified rudder is a very slender object with low frontal resistance. On the other hand, resistance should be evaluated together with the propulsion capacity.

The handbook on practical ship hydrodynamics states that propulsion and resistance are complex problems, and to solve them a CFD analysis is preferred [2]. In ship hydrodynamics theory, rudder is one of the appendages. The resistance of the appendages under service conditions is tested separately or not tested at all. If the appendage size is small compared to the ship, one would expect an error in resistance calculation to be small. The chapter "Resistance and Propulsion" of the book [2] states that the significance of the resistance created by appendages depends on their size compared to the ship size. Thus, the more complex appendages are installed on the floating object, the more important it is to evaluate their resistance. For the CFD study on submarines, Shariati et al. showed that the total resistance of a submarine increased by fitting appendages on it [3]. The authors used Star CCM+ software, developed using a finite volume method. In that study, the major influence of appendage resistance was detected on the viscous part of the total resistance, while wave making resistance was not affected significantly [3].

The SUP board is a small craft compared to ships. As noted by Carlton [4], for small craft propellers are usually considered a simpler and lighter propulsor. However, in this project a waterjet propulsor was chosen as a safer option compared to an open shaft propeller. Since a SUP board for tourism is mainly used on the rivers, a propulsor needs to be protected from the water plants. Sometimes the bottom of the board is floating very close to the underwater stones. Thus, the case or duct of the waterjet is supposed to be the barrier, which saves propulsion from potential damage. Propeller could still be used as a SUP board propulsor; however, a duct is needed to protect it. In fact, Cooper et al. [5] suggest that a waterjet could be considered a special type of ducted propeller. Majority of test procedures for waterjet propulsion and propellers are currently the same [6].

The typical SUP board weights around 13-14 kg. Depending on the style and size, there could be lighter versions starting from $7 \mathrm{~kg}$ and some as heavy as $18 \mathrm{~kg}$. The added mass of a propulsion system should be small enough to ensure that buoyancy is not disrupted.

The waterjet is attached to the SUP fin (rudder) in the following way: the fin is cut into two parts, and the waterjet is in between - far enough from the SUP board to provide space for the charging battery. The waterjet case is a barrier for water plants, fish and stones. As a negative aspect, it is a barrier to the water as well. As noted in the review paper [7], efficient inlets for the waterjet are crucial for the successful performance of the system. The task is, therefore, to choose 
the design option balancing between the ease of manufacturing and efficiency.

In this study, we investigated the drag force exerted on the appendage using SolidWorks Flow Simulation software. Two types of simulations (type 1 - the pressure on the body; type 2 - the flow around the body) were tested and compared for accuracy and computational time. Albeit computer simulations are time consuming, the importance of CFD modelling specifically for waterjets is stressed in the Procedures of the Specialist Committee on Validation of Waterjet Test [7]. The Specialist Committee published a review on updated literature about Intake Design and Analysis [7]. The authors in [7] quoted the analysed waterjet inlets for large watercrafts, yet their research stressed the importance of CFD simulations and provided guidance on proper boundary conditions to be applied to a model.

Bulten in his $\mathrm{PhD}$ thesis [9], quoted in reference book [4], compared numerical methods used by waterjet industry leaders Wärtsilä Propulsion Netherlands BV and he found that the simplified method to describe waterjet installations was not cor- rect because existing computer programs did not calculate the influence of the hull in the vicinity of the waterjet inlet and the pressure distribution acting on the stream tube [9].

The hypothesis of this study stated that optimising the shape of the waterjet would improve the efficiency of the proposed propulsion solution. The hypothesis was based on the theory provided by Hoerner [10]. Hoerner showed that rounding-off edges in blunt shapes was not perfect (compared to a complete streamlining procedure) but still efficient method to reduce a drag coefficient. Results section showed that this hypothesis was true. Rounding-off edges provided $35 \%$ of drag coefficient reduction, and additional reduction of $10 \%$ was obtained after applying a streamlining solution.

Waterjet in operation induces reaction forces and moment and waterjet/hull interaction stern force [11]. These forces are out of scope of this research paper, but the velocity effect will be discussed in this paper.

\section{THEORY}

\section{Forces Acting on the Body}

According to the goal of this study, the hydrodynamic resistance of the modified rudder should be minimised to ensure propulsion efficiency. In this study, we minimise it by reducing the drag force.

Following the equation from fluid mechanics fundamentals on the external flow [12], the drag force acting on a body is given by Eq. (1):

$F_{D}=\frac{1}{2} \rho V^{2} C_{D} A$, where $F_{D}-$ drag force, $N$ or $\mathrm{kg} \cdot \mathrm{m} / \mathrm{s}^{2} ; \rho-$ fluid density, $\mathrm{kg} / \mathrm{m}^{3} ; V$ - relative velocity, $\mathrm{m} / \mathrm{s} ; C_{D}$ - a drag coefficient, dimensionless; $A$ - a frontal area of the body, $m^{2}$. The term $\frac{1}{2} \rho V^{2}$ is called dynamic pressure [12].

Figure 1 demonstrates the forces acting on the SUP board and a person standing on it. The SUP board, a person standing on it and a paddle a person holds are moving in two media - the water and the air, respectively. 


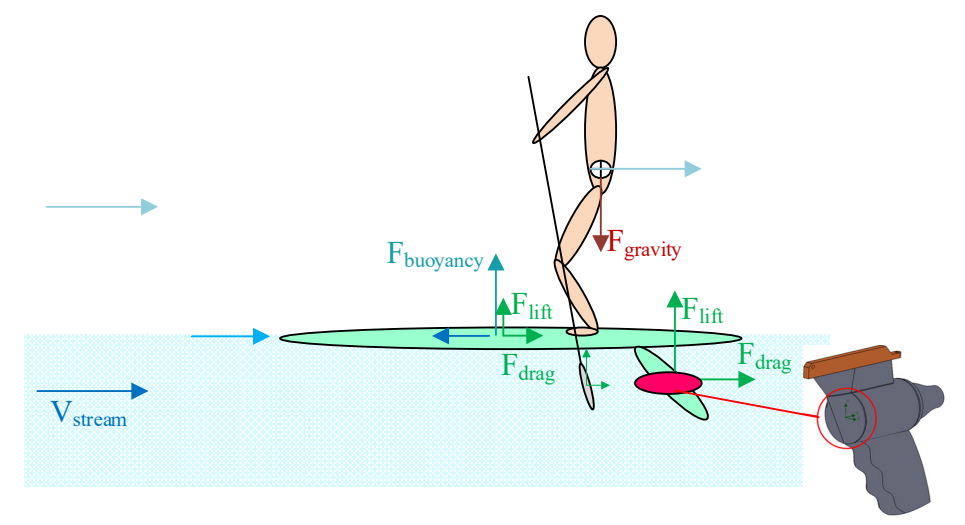

Fig. 1. The forces acting on the SUP board (green colour), the person driving the board (skin colour) and SUP board appendage, including the waterjet (red colour) attached.

According to Eq. (1), the drag force depends on the frontal area of the body. Figure 1 suggests that the area of the modified rudder (red coloured area) would be small compared to the area of the person (skin coloured area). On the other hand, water density is more than 800 times greater than the air density [12]. Therefore, the appendage under water increases the total drag force of a SUP board more than the appendage above the water would do. It is also desirable to minimixe the drag force for the appendage because it would lessen the stresses on the attachment of the SUP fin to the board. We acknowledge that manufacturers of SUP boards do not design the attachment of the fin to experience great frontal drag force, and therefore knowledge about the drag force experienced by appendage is of special importance.

In the water medium, the hydrostatic pressure and resulting buoyancy force are dominant. The hydrodynamic drag and lift forces are acting on a board, but for such a slender body as a SUP board the shear

\section{Waterjet Design}

According to the handbook on propulsion systems, the waterjet design could be simplified to three main components: an inlet ducting, a pump and an outlet or nozzle [4]. force acts nearly parallel to the flow direction, and thus their contribution to the lift is small [12]. The drag force for such a slender body is mostly due to the skin friction drag, which is tangential force [10] and is also relatively small. Therefore, for the streamlining purposes we will analyse the hydrodynamic pressure locally on the fin, where the waterjet is attached (see the red circle on Fig. 1). Results show that adding a waterjet duct to the fin increases fivefold the drag force a flowing fluid exerts on a body in the flow direction.

The part of resistance force that is related to pressure is called the pressure drag or form drag because of its strong dependence on the form or shape of the body [12]. The pressure field of the SUP board causes deformation of water surface, and a wave system originates behind the board. The wave making resistance force is acting normal to the body as shown in Fig. 1. Since we focus on the drag force of the immersed part, wave making resistance is out of scope in calculations.

The physics of waterjet propulsion is described in reference book [13]. It states that water is drawn through a ducting system by an internal pump. The pump adds 
energy to water and it is expelled from the ducting system at high velocity. The unit thrust is primarily generated as a result of the momentum increase imparted to water [13]. The mathematical expression for the description above is provided by Carlton [4] as shown in Eq. (2):

$T=\rho A_{2} V_{2}\left(V_{2}-V_{1}\right)$,

where $T$ - propulsor thrust produced by the system, $N ; \rho$-density of the water, $\mathrm{kg} / \mathrm{m}^{3}$; $A_{2}$ - a cross-sectional area of the nozzle, $\mathrm{m}^{2}$; $V_{2}$ - velocity of the water leaving the system, $m / s ; V_{1}-$ velocity of the water entering the system, $m / s$.

\section{Inlet Velocity Ratio (IVR)}

The Specialist Committee on Validation of Waterjet Test Procedures [7] suggests using abbreviation IVR (Inlet Velocity Ratio) when describing intake working point.

The IVR is calculated according to Eq. (3) [4]:

$\operatorname{IVR}=\frac{\mathrm{V}_{1}}{\mathrm{~V}_{\mathrm{s}}}$,

\section{Boundary Layers}

A boundary layer is a thin layer of viscous fluid close to the solid surface of a wall in contact with a moving stream. The flow velocity at the wall is zero [10], since the flow "sticks" to the wall because of its viscosity.

According to Ledoux et al. [14], jets form "a special group of mixing boundary layers" because there are an outer boundary layer and an inner boundary layer, and therefore Ledoux et al. [14] suggest using a numerical approach for jet problems.

The behaviour of differently shaped objects in the flow was presented by Vutukuru et al. [15]. The authors used ANSYS
No matter how efficient the internal pump is, there are going to be some performance losses caused by flow distortion on the intake. To minimise inlet losses, this paper focuses on the inlet design. The inlet ducting consists of tunnel, inlet and supporting structures [4]. Equation (2) suggests that achieving more thrust requires greater difference between the water inlet and outlet speed. Thus, outlet nozzle should be designed to produce a greater speed of the water leaving the system, while the inlet design, on the contrary, should ensure the water entering speed to be possibly close to the floating object speed. To describe the inlet efficiency, the term "inlet velocity ratio" is commonly used.

where $V_{1}$ - the water inlet velocity, $\mathrm{m} / \mathrm{s} ; V_{s}-$ the craft velocity, $\mathrm{m} / \mathrm{s}$.

Following Eq. (3), as the water inlet velocity decreases due to some constructive barrier, the IVR decreases and the flow rate through the waterjet decreases.

software to study the interaction forces for the five different forms of the prism, and "boundary layers set to stair stepping with maximum of 7 layers" were used for each shape. The results of the study suggested that the pressure in the suction zone along the boundary layer was independent of the shape of the object with sufficient accuracy (about $2 \%$ ), and one could assume "a constant pressure in the boundary layer of the flow suction zone at low speeds" [15]. Considering the results above, in this study we focus on the resistance independent of the boundary layer. 
One of the goals of the project is to develop a full-scale prototype of the waterjet using available 3 -dimensional (3D) printing techniques and to test it in the water. Meanwhile, before the optimal design parameters are known, the tests were performed using SolidWorks Flow simulation software. The 3D simulation, performed in SolidWorks Flow Simulation, discretizes the timedependent Navier-Stokes equations and solves them on the computational mesh. More details are provided in the section "Mesh Size".

The simulation to obtain a drag coefficient for the rudder with the waterjet attached was performed twice; namely, the simulations of two types were completed. The first type (further referred to as Type 1) was the simulation of the pressure on the body. This simulation focused on the pres- sure zone on the appendage. The pressure is simulated as a vector acting on the frontal area. The second type of the simulation (further referred to as Type 2) was simulation of the flow around the body, and it focused on the pressure distribution on the whole surface of the object (the uniform body). The convergence goal (a variable to calculate from the fluid flow) was the force (see Eq. (1)). The initial condition of fluid flow inlet velocity was specified to be $2.5 \mathrm{~m} / \mathrm{s}$. It was expected that the simulation of Type 2 would provide higher precision. However, we found out that for the specific study of a drag coefficient, results of both simulations were very close, while simulation of Type 1 provided results faster, and the results were easier to analyse. Detailed comparison is provided in the Results section.

\section{Rounding-off Edges and Streamlining}

The waterjet cover and rudder are optimised in SolidWorks software to reduce resistance force from the fluid flow. The part subjected to analysis was an intake chamber - motor cover as shown in Fig. 2.

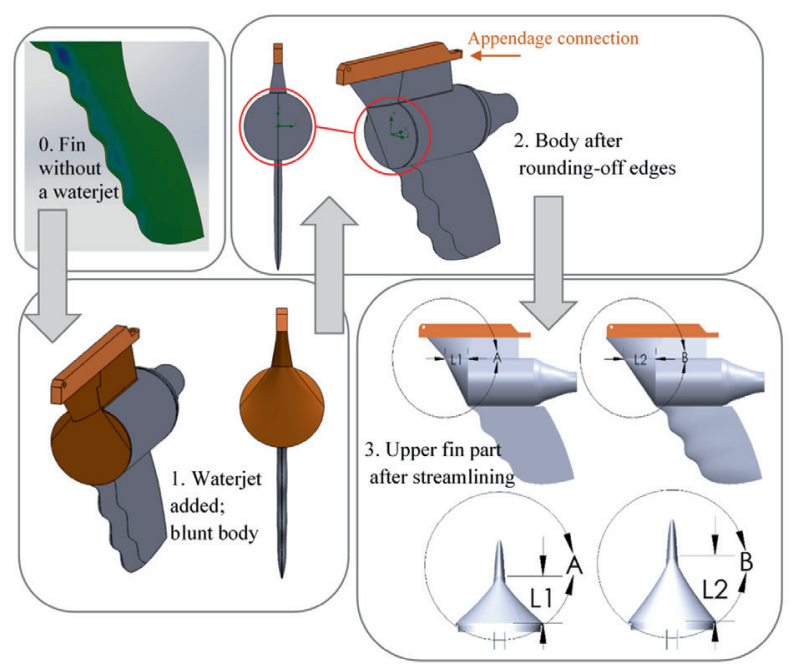

Fig. 2. The fin and waterjet view before modifications and after rounding-off edges and streamlining in SolidWorks. 
Figure 2 shows the sequence of the modifications made to the fin and the optimised design of the waterjet. The area marked by red circle shown on the appendage is further streamlined - for the chosen optimal design of the fin, the drag coefficient for different fin lengths (from L1 to L2) is studied. The difference in fin length is demonstrated in Fig. 2 as circle A and circle B. In circle A, the fin is shorter than appendage connection (L1), in circle B the

\section{Mesh Size}

For meshing the model, SolidWorks Flow Simulation uses a technique called the Finite Volume Approach. For the simulation of fin without a waterjet, the generated mesh consisted of $2.76 \cdot 10^{5}$ cells, for a simu- length of the fin is prolonged to the maximum value that appendage connection permitted (L2 = L1 + $30 \mathrm{~mm})$. For the streamlining study, the simulation of flow pressure was performed with a step (difference in fin length) of $5 \mathrm{~mm}$, starting with $\sim 0 \mathrm{~mm}$ (L1) and up to $30 \mathrm{~mm}$ (L2). The results of the drag coefficient dependency on rudder length are shown in Fig. 7 (simulation of Type 1).

lation with a waterjet - it contained more than $7 \cdot 10^{5}$ cells. The local mesh is intended for resolving the mesh around the highest pressure in the frontal cylindrical region. An example of the mesh is shown in Fig. 3.

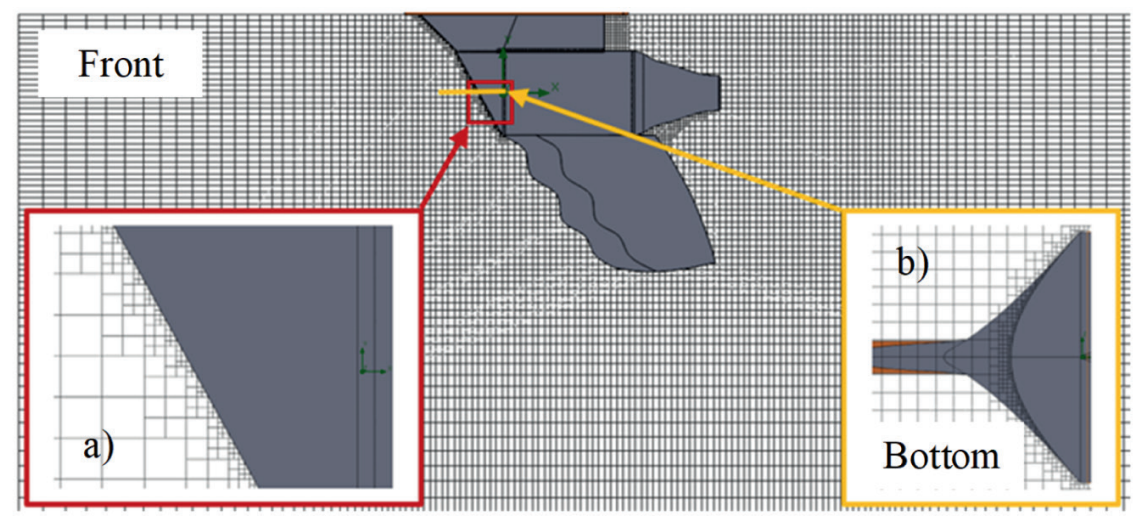

Fig. 3. The illustration of the mesh size.

Figure 3 provides the example of the front cut plot of initial global mesh domain with refinement level four (4), zoomed view for local mesh at cylindrical part in front (red frame-a)) and bottom (yellow frame-b)) planes.

\section{RESULTS AND DISCUSSION}

The three-dimensional finite element simulation results are given in this section to illustrate calculation of drag coefficient.
Figure 4 shows the selected results from the simulation of Type 1 (the pressure on the body). 

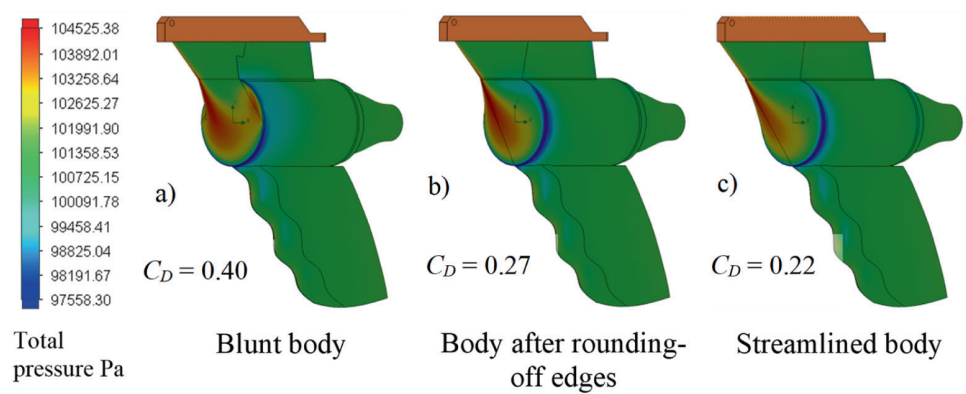

Fig. 4. The pressure on the front surface and resulting drag coefficient for original blunt body, body after round-off edges and streamlined body.

The example of results for the simulation of Type 2 (the flow around the body) is the flow around the body is available congiven in Fig. 5. The complete animation for tacting the corresponding author.

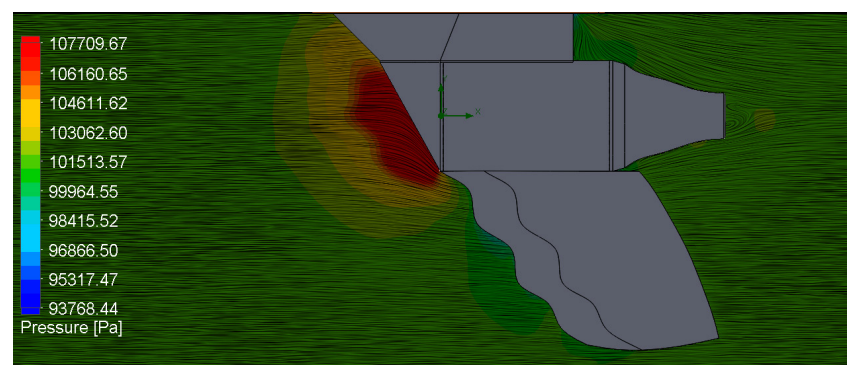

Fig. 5. The example of the simulation of the flow around the body. Frontal pressure distribution and streamlines.

\section{Drag Coefficient and Resulting Force}

Simulation of the pressure on the body showed that the drag coefficient for the fin without a waterjet attached was only 0.078 . Drag coefficient increased to 0.4 as the waterjet was added.

The results obtained from simulations for the fin with a waterjet attached are summarised in Fig. 6, where the design of blunt body (Fig. 4a) is indicated with orange markers, and the improved design of streamlined body is indicated with blue markers. The second-order polynomial approximation is used for the streamlined body results.

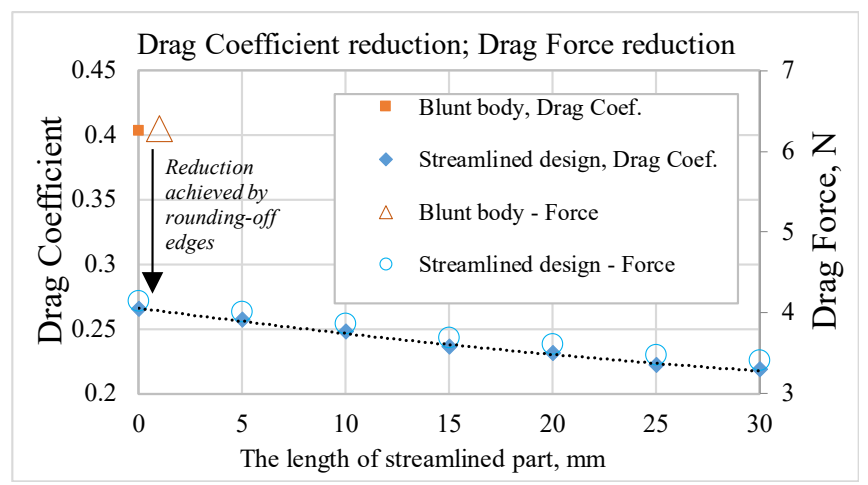

Fig. 6. Drag coefficient and drag force reduction after rounding-off the edges of the waterjet duct. 
Figure 6 shows that rounding-off the edges of waterjet duct reduces the drag coefficient from 0.4 to $0.27-0.22$ depending on the length of the appendage. It means that the reduction of drag from $35 \%$ to $45 \%$ is achieved, so rounding-off edges is strongly suggested during manufacturing. Meanwhile, further streamlining of design results in $10 \%$ reduction of drag coefficient (see Fig. 2 for illustration of difference between the appendage length from L1 to L2 $=30$ $\mathrm{mm}$ ), so it is recommended to apply it, if manufacturing capacity permits. Figure 6 shows a summary of the average drag coefficient, obtained from the simulation of 200 iterations in SolidWorks software. Please refer to Fig. 7 for a complete illustration of drag coefficient simulation.

According to Fig. 6, the average drag coefficient and drag force decrease as the fin length increases, but for the fin length, which equals $30 \mathrm{~mm}$ (this limit is set by the size of the attachment), the gained decrease is almost equal to design of $25 \mathrm{~mm}$, so the further streamlining attempts would be less efficient.

\section{Comparing the Drag Coefficient Results from Two Simulation Methods}

In this study, two types of simulations were performed to predict the drag coefficient. The first type of the simulation was for the pressure on the body. The second type of the simulation was for the flow around the body. The results slightly differed for both methods because for the simulation of Type 2 on the flow around the body the mesh size was smaller. Figure 7 illustrates the difference of simulation results for the drag coefficient change during the simulation of the pressure and simulation of the flow.

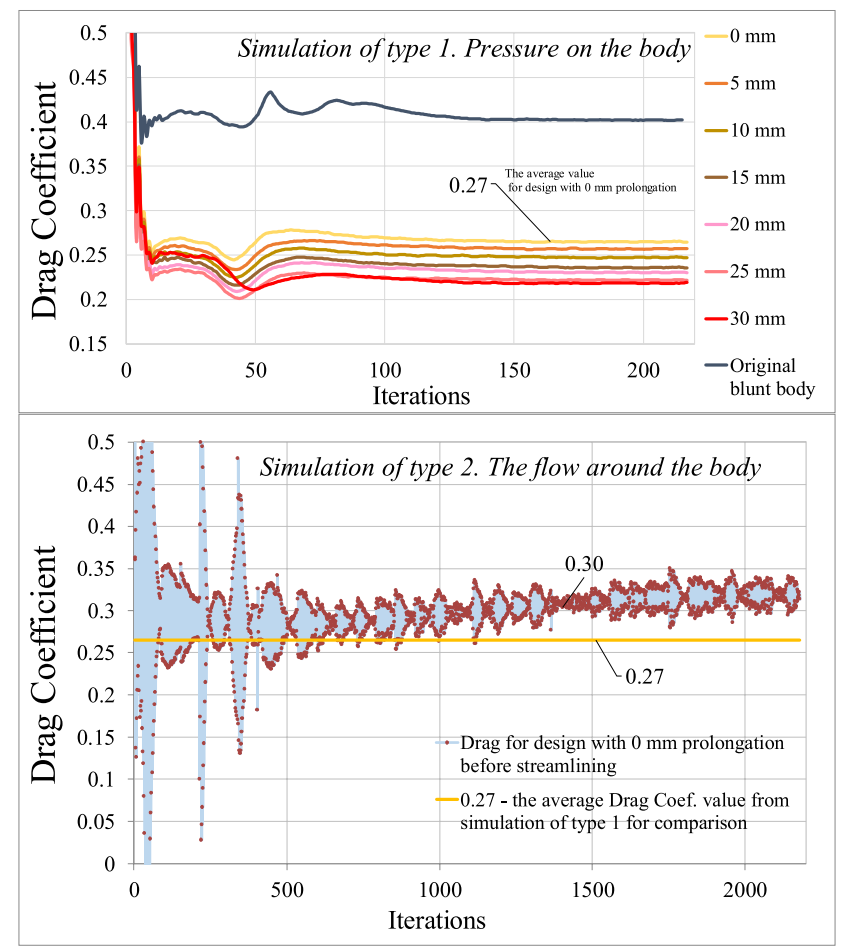

Fig. 7. Drag coefficient values from simulation results for each iteration in SolidWorks - comparison of the drag coefficient values calculated from pressure and from the flow around the body. 
To compare measurement results from the two simulations, zeta-scores test [16] for the design of $\sim 0 \mathrm{~mm}$ prolongation (no streamlining) was performed. For the simulation of Type 1 (the pressure on the body), the mean value (from all iterations of the simulation, including instability region) of the drag coefficient was 0.27 , while for the simulation of Type 2 (the flow around the body) the mean value was 0.3 . Uncertainty at standard deviation level, , was calculated to be 0.08 and 0.11 for simulation of Type 1 and Type 2, respectively. The absolute value of the zeta was calculated to be 0.22 . It is below 2, which means that the results are not in disagreement. From this analysis we concluded that the simulation of Type 1 should be preferably used to explore different design options because it required less time to complete. For comparison, the simulation of Type 2 takes around 17 hours on a standard laptop computer, while simulation

\section{Inlet Location}

After the study of uniform body is completed, the analysis of the best inlet position could be discussed. According to Eq. (3), the inlet should be located to allow for maximum water inlet velocity. The design that is easy to manufacture requires water entering the jet through the grooves cut on the side of the tunnel. This design has a disadvantage - the flow from the sides is small compared to the flow from the front plane. The dark blue colour on the sides of the tunnel in Fig. 4 indicate that the pressure on the of Type 1 could be completed in a couple of hours. Thus, for the study of the drag coefficient the simulation of pressure is explicit enough. The simulation of Type 2 on the flow around the body should be used when more information about the changes of the drag coefficient in time is needed.

The data in Fig. 7 suggest that during the simulation of the flow around the body the following phenomena occur: as the flow first meets the rudder, the drag coefficient is larger, then it drops to its lowest value, but then it remains stable.

The simulation of the flow around the body could later be used for further studies of vortexes around the body. The flow simulation is also helpful to describe the cavitation effects that may occur in the pump or in the intake during operation of the vessel. Cavitation does not affect the powering characteristics, but it causes possible erosion effects [7].

sides is the smallest; therefore, the amount of water entering the inlet from the side will be much smaller than the amount of water entering the inlet from the front.

Therefore, we propose creating the waterjet inlet at the front of the tunnel, and in order to protect the various internal waterjet components, to fit it with a guarding mesh, keeping in mind the following principle - the finer the guarding mesh of the inlet, the better protection.

\section{The Velocity Effect on the Drag Coefficient}

For the purpose of simulation, the velocity was estimated to be $2.5 \mathrm{~m} / \mathrm{s}$ or $9 \mathrm{~km} / \mathrm{h}$, which resulted from the estimation of SUP board moving speed. According to the external flow theory, the drag coefficient depends on the Reynolds number, specifically below $10^{4}$. At higher Reynolds numbers, the drag coefficients is expected to remain constant [12]. In this study, Reynolds number was calculated to be $\mathrm{Re}$ $\sim 475000$; therefore, the obtained drag coeffcient would be similar for moving speeds that differed from the assumed speed. When the waterjet is in operation, the speed of the 
board increases, but given the Reynolds number calculated the drag coefficient will not differ significantly.

\section{CONCLUSIONS}

1. Simulation results showed that waterjet added to the rudder accounted for significant frontal resistance. The drag coefficient of the rudder without a waterjet was only 0.078 . It increased to 0.4 as the waterjet was added. Drag coefficient was reduced to 0.23 by choosing the optimal design of the waterjet duct.

2. Rounding-off the edges of waterjet duct accounted for $35 \%$ of drag coefficient reduction compared to the initial blunt design, so this improvement was worth applying the necessary manufacturing effort. The further streamlining accounted for additional $10 \%$ decrease in drag coefficient. In total, $45 \%$ of drag coefficient could be obtained by working on the design of the waterjet frontal plane.
3. The study showed that predicting the drag coefficient for the appendage in water could be done via two types of the simulation in SolidWorks Flow. The first type of simulation was the pressure on the body. The second type of simulation was the flow around the body. Both simulations provided clear results and the trends were in a good agreement. The results slightly differed because for the simulation of Type 2 the mesh size was smaller. Simulation of Type 1 required less computational time and was therefore preferable for the study of the drag coefficient. Simulation of Type 2 is preferred when the drag change in time and vortices around the body should be studied.

\section{ACKNOWLEDGEMENTS}

The research has been conducted within the project "Development of Technical Solutions for Water Transport Transmission System", grant number ZI-2020/3, Frame- work of Science and Innovation within research platforms at Riga Technical University, funded by Riga Technical University.

\section{REFERENCES}

1. Jennings, G. (2007). Motorized water sports, water-based tourism, sport, leisure, and recreation experiences. USA: Elsevier.

2. Bertram, V. (2000). Practical ship hydrodynamics. UK: Butterworth-Heinemann.

3. Shariati, S. K., \& Mousavizadegan, S. H. (2017). The Effect of Appendages on the Hydrodynamic Characteristics of an Underwater Vehicle near the Free Surface.
Applied Ocean Research, 67, 31-43. DOI: 10.1016/j.apor.2017.07.001

4. Carlton, J. (2018). Marine propellers and propulsion. USA: Butterworth-Heinemann. DOI: 10.1016/B978-0-08-100366-4.00016-X

5. Cooper, R. D., \& Doroff, S. W. (1971). Unsteady propeller forces, fundamental hydrodynamics and unconventional propulsion. Rome, Italy: Office of Naval Research. 
6. Gong, J., Guo, C. Y., Wang, C., Wu, T. C., \& Song, K. W. (2019). Analysis of Waterjet-Hull Interaction and its Impact on the Propulsion Performance of a Four-Waterjet-Propelled Ship. Ocean Engineering, 180,211-222. DOI: 10.1016/j. oceaneng.2019.04.002

7. Brandau, J. H. (1968). Performance of Waterjet Propulsion Systems - A Review of the State-of-the-Art. Journal of Hydronautics, 2 (2), 61-73. DOI: 10.1109/ULTSYM.2013.0335

8. Specialist Committee on Validation of Waterjet Test Procedures. (2005). Final Report and Recommendations to the 23rd ITTC. In Proceedings of 24th International Towing Tank Conference, (pp. 387-415), 26 March 2002, Edinburgh, Scotland: The University of Newcastle.

9. Bulten, N. W. H. (2006). Numerical analysis of a waterjet propulsion system. Eindhoven, the Netherlands: Technische Universiteit Eindhoven. DOI: 10.6100/IR614907

10. Hoerner, S. F. (1965). Fluid dynamic drag: Practical information on aerodynamic drag and hydrodynamic resistance. Washington, D.C., USA: Hoerner Fluid Dynamics.

11. Kandasamy, M., Ooi, S. K., Carrica, P., \& Stern, F. (2010). Integral Force/Moment Waterjet Model for CFD Simulations. Journal of fluids engineering, 132 (10), 101103-101112. DOI: 10.1115/1.4002573
12. Cenqel, Y. A., \& Cimbala, J. M. (2017). Fluid mechanics: Fundamentals and applications $\left(4^{\text {th }}\right.$ ed). New York, USA: McGrawHill Education.

13. Molland, A. F. (2011). The maritime engineering reference book: A guide to ship design, construction and operation. Hungary: Elsevier.

14. Ledoux, M., \& Hami, A. E. (2017). Compressible flow propulsion and digital approaches in fluid mechanics. UK: Wiley-ISTE.

15. Vutukuru, S. K., Tipans, I., Viba, J., \& Irbe, M. (2020). Form optimization and interaction analysis of plane symmetry prism in air. In the 19th International Scientific Conference "Engineering for Rural Development" (pp. 739-746), 20-22 May 2020, Jelgava, Latvia: Latvia University of Life Sciences and Technologies. DOI: 10.22616/ERDev2020.19.TF170

16. University of Tartu. (2013). Lecture 12. Comparing Measurement Results Using Measurement Uncertainty Estimates. Available at https://sisu.ut.ee/measurement/12using-measurement-uncertainty-estimatesdecision-making 